\begin{tabular}{|c|c|}
\hline Title & Continuous wave al exandrite laser pumped by a direct-current mercury arc lamp \\
\hline Author(s) & Torizuka, Kenji; Y amashita, Mikio; Y abiku, T sukasa \\
\hline Citation & $\begin{array}{l}\text { A pplied Optics, 32(36), 7394. } 7398 \\
\text { https://doi.org/10.1364/A 0.32.007394 }\end{array}$ \\
\hline Issue Date & $1993-12-20$ \\
\hline Doc URL & http:/hdl.handle.net/2115/45324 \\
\hline Rights & (1993 Optical Society of A merica \\
\hline Type & article \\
\hline File Information & А 0 32-36_7394 7398.pdf \\
\hline
\end{tabular}

Instructions for use 


\title{
Continuous-wave alexandrite laser pumped by a direct-current mercury arc lamp
}

\author{
Kenji Torizuka, Mikio Yamashita, and Tsukasa Yabiku
}

\begin{abstract}
A fluctuation-free mercury arc lamp for the pumping of a cw alexandrite laser is reported. Thermal insulation of a cathode of the lamp permits $4-\mathrm{kW}$ fully vaporized direct-current operation. Fluctuations owing to the pool of liquid mercury in conventional lamp tubes is completely avoided. A $17-\mathrm{W}$, cw alexandrite laser pumped by the newly developed lamp is described.
\end{abstract}

\section{Introduction}

Alexandrite is a unique tunable laser material that will lase continuously with arc-lamp pumping. ${ }^{1-5}$ However, such a lamp-pumped cw laser has not yet been in practical use because of the following problems with the pumping sources and the xenon or mercury arc lamps: First, during xenon arc-lamp pumping, the transmission of the output coupler $\Gamma$ is very small $(\Gamma \lesssim 1 \%)$ because of mismatching between the absorption spectrum of alexandrite and the emission spectrum of the lamp, even though the lamp operates with good stability. Second, although highpower mercury arc lamp provides a more efficient pumping source that allows $\Gamma$ of $3-4 \%,{ }^{2}$ this type of lamp is usually unstable in direct-current (DC) operation owing to the existence of liquid-phase mercury in the lamp tube.

In this paper, we describe a stable DC mercury arc lamp used to pump an alexandrite laser. A newly designed cathode for the mercury arc lamp enables us to achieve fully vaporized operation with $4-\mathrm{kW}$ input power over a 70-h (typical) lamp life. Using the newly developed lamp, an $\sim 17-\mathrm{W}$ cw laser output with an $\sim 0.7 \%$ slope efficiency is achieved without screening of the alexandrite rods.

When this research was performed K. Torizuka and M. Yamashita were with the Laser Section, Opto-Electronics Division, Electrotechnical Laboratory, 1-1-4 Umezono, Tsukuba, Ibaraki 305, Japan; and T. Yabiku was with the Lamp Division, ORC Manufacturing Company, Ltd., 3-34-1 Chofugaoka, Chofu, Tokyo 182, Japan. M. Yamashita is now with the Faculty of Engineering, Hokkaido University, Kita-13-Nishi-8, Kita, Sapporo, Hokkaido 060, Japan.

Received 25 January 1993.

0003-6935/93/367394-05\$06.00/0.

(1) 1993 Optical Socicty of $\Lambda$ morica.

\section{Experiment}

\section{A. Design and Performance of Lamps}

A conventional high-power mercury arc lamp contains liquid mercury even when it is in operation. As a result of the thermal balance achieved between heating by lamp discharge and cooling by the water, the liquid mercury pools at the low-temperature area around the electrodes. Two electrodes of the lamp are the same in shape because they are designed for alternating-current (AC) operation. However, when the arc lamp is operated in the DC mode, the heat generated at the anode is much greater than that generated at the cathode. Thus, mercury condenses and vaporizes randomly around the cathode, consequently producing serious fluctuations in lamp emission.

To develop a new cw mercury arc lamp, we at first examined the relation between the amount of liquid mercury in the lamp tube and the emission stability. We found that reducing the amount of liquid mercury surrounding the electrodes improved the stability during DC operation to some extent. However, we could not avoid completely the above-mentioned fluctuation when the amount of mercury in the tube was more than optimum. When the amount of mercury in the tube was less than optimum, the arc pressure became too low, and, the supplied current necessary for efficient lasing became so high that the lamp broke. Even with finely adjusted optimal amounts of mercury, the conventional lamp tube was stable over only a narrow range of supplied power. The stable range, furthermore, each varies severely from lamp to lamp and drifts during the lamp operation.

Therefore, we concluded that realizing fully vaporized operation with stability over a wide range of supplied power is one way to improve the lamp. To 
obtain reliable DC lamp operation with sufficient power for a cw alexandrite laser, we carried out the following investigations: optimization of the thermal insulation of a cathode to prevent mercury condensation, and adjustment of the amount of mercury in the lamp tube.

\section{Lamp Structure}

Figure 1 shows the structure around the electrodes of the lamps schematically. An electrode of a typical conventional lamp is shown in Fig. 1(a), and the anode and cathode of the lamp we developed are shown in Figs. 1(b) and 1(c), respectively. Both lamps have additional ceramic sleeves to be held in the pumping chamber. All of the electrodes are sealed using the familiar molybdenum-foil techniques. In each end of the conventional lamp, a pin-type electrode is used, and it becomes wet with the liquid mercury from the pool surrounding the base of the electrode. In the lamp we developed for DC operation, we needed to use different structures in both the anode and cathode. The anode is strongly heated, so we prevented the mercury from condensing by cooling the tube wall with flowing water Fig. 1(b). To

(a)

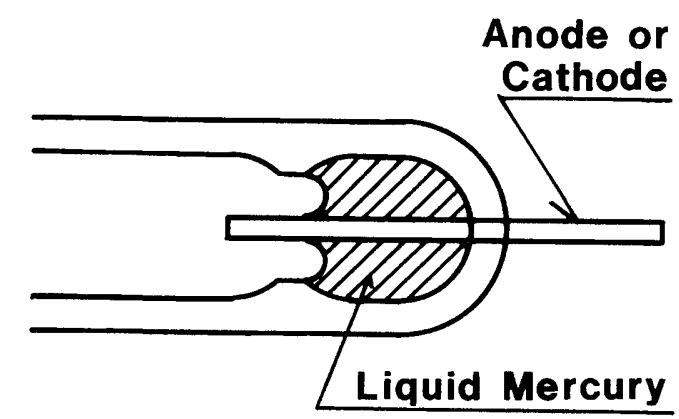

(b)

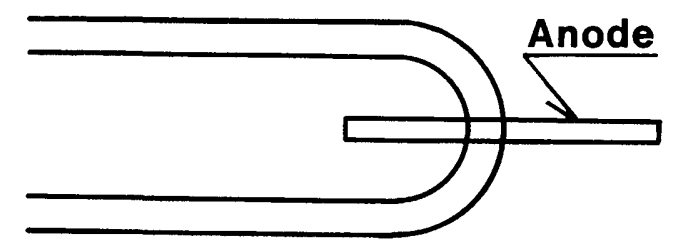

(c)

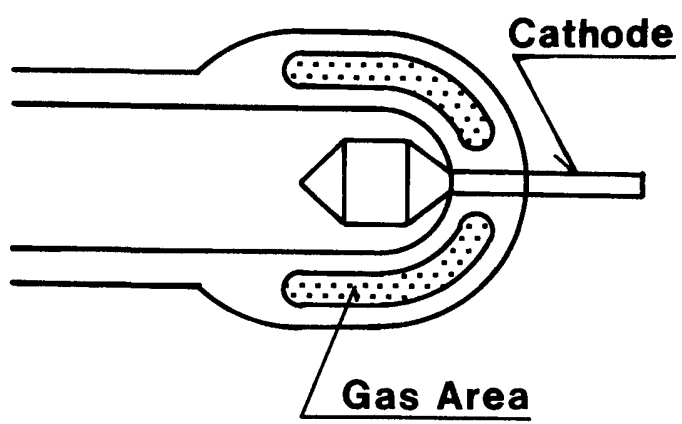

Fig. 1. Schematic diagram of an electrode of (a) a conventional mercury arc lamp, and (b) the anode and (c) the cathode of the newly developed lamp. minimize the occurrence of cracks caused by thermal stress around the anode, we found that a thinner electrode works better; we used a tungsten electrode with a diameter of $\sim 0.6 \mathrm{~mm}$ and achieved $200-300 \mathrm{~h}$ of anode life. Around the cathode, thermal shielding from the cooling effects of the flowing water is necessary to prevent mercury condensation. Too much insulation, however, can shorten the lamp life because of the thermal stress. Thus, we used a concentric double-walled tube around the cathode to shield it; the gap between the walls is filled with a noble gas. In the early stage of development, the cracks around the cathode were the main problem preventing lengthening of the lamp life. The occurrence of cracking is much decreased if the gas is contained in what we found to be the optimum shape, a tapered cylinder. Our cylinder was $15-\mathrm{mm}$ in length. The walls of the gas cylinder cover the entire electrode, and wall thickness is kept as uniform as possible. The electrode is made of tungsten and is 9 $\mathrm{mm}$ in length, $2.5 \mathrm{~mm}$ in maximum diameter, and tapered to both ends [see Fig. 1(c)], where the one end is a cone for the arc and the other is connected with a molybdenum foil.

The mercury pressure in a conventional arc lamp is dependent on the heat generated at the top of the electrode and is balanced by the thermal conduction of the electrode. When the supplied current increases, the vaporization of mercury in the pool is enhanced. Therefore, the mercury pressure increases steeply with increases in supplied power, owing not only to the temperature of the arc but also to the amount of vaporized mercury. The range of the supplied power one can use is narrow because increasing pressure breaks the lamp. During fully vaporized operation, the mercury pressure is dependent on only the arc temperature because the amount of vaporized mercury is constant. Operation then is possible over a relatively wir.e range of supplied power. However, a critical adjustment of the amount of mercury is necessary to control the mercury pressure during operation. The amp we developed is made so that the mercury pressure is approximately 30-50 atm. The emission intensity, matched with the absorption band of alexandrite, is checked with a photometer. Although the mercury pressure of the lamp we developed is lower than that of a conventional AC lamp, the emission intensity is as strong as that of the conventional lamp because the supplied power density is relatively high.

We also found that conventional lamps that operated outside the pumping chamber with good stability and life length break down quickly when operated inside the chamber. This fact suggests that lamp emission returned from the pumping reflector further increases the lamp temperature. In our development, therefore, the cathode structure is optimized for use in a pumping chamber. 


\section{Lamp Performance}

With the improvements mentioned in Section 2.1, we found that more than $90 \%$ of the lamps we manufactured survive for $30-100 \mathrm{~h}$ during $4-\mathrm{kW}$ operation. No changes in lamp emission are observed even after the long operation. Each lamp endures being turned off and on 30-100 times during its lifetime. The lamps break down when cracks form around the cathode that are caused by the high-voltage trigger used to start lamp discharge, and the lamp does not explode. The lamp we developed is stable during operation with supplied power of $\geq 2 \mathrm{~kW}$, and it is free from mercury condensation. ${ }^{3}$ For supplied power of $\leq 1.5 \mathrm{~kW}$, however, the arc fails.

The newly developed lamp is driven by a powerstabilizing high-voltage supply with a maximumcurrent limiter. To decrease misfiring of the arc, the lamp is triggered with an ignition coil, and it is initially operated with a maximum current of $4 \mathrm{~A}$ for several seconds until the supplied power rises to a preset value. In steady operation with supplied power of $2-4 \mathrm{~kW}$, the voltage is in the range of $1.2-1.5$ $\mathrm{kV}$. Figure 2 shows the typical rise in emission intensity obtained from different lamps with various amounts of supplied power. The vertical dashed lines indicate the starting time. In repetitive measurements of one lamp, emission-intensity curves are not always the same. We consider that the differences in emission-intensity rise-up curves are caused mainly by variation of the initial distribution of liquid mercury. Note that in most cases, the emission intensity becomes steady within 1 min. After the rise-up transiency is complete, nearly equal conversion efficiencies from supplied power to emission are obtained in all the lamps. No flickers of emission are observed in the lamps we developed.

The outer and inner diameters of the lamp tube in the discharge area (which is $76 \mathrm{~mm}$ in length) are 5 and $2.7 \mathrm{~mm}$, respectively. The maximum outer diameter, however, is $7 \mathrm{~mm}$ at the cathode because of the

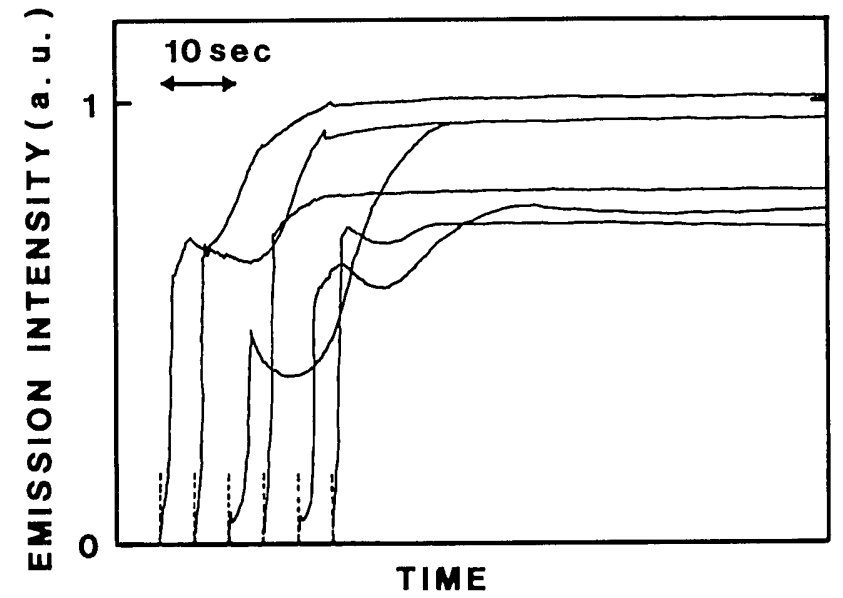

Fig. 2. Rise in emission intensity for six samples obtained with the newly developed DC lamp driven by the same power supply (in text). The emission obtained during steady-state conditions is nearly proportional to the supplied power. double-walled tube providing thermal insulation. The lamp is operated in a Pyrex tube (9.6-mm inner diameter) while keeping a flow of deionized water (7-L/min flow rate).

\section{B. Cw Laser Experiments}

We designed a pumping chamber for a cw alexandrite laser for the arc lamps we developed. The pumping chamber contains a double-ellipse reflector with 39.6and 42.6-nm diameters and a $15.5-\mathrm{mm}$ focal point separation that equals the distance between the lamp and the laser rod. Continuous lasing of alexandrite has been carried out using reflector coatings of electroplated silver, evaporated aluminum, or evaporated silver on the elliptical cylinder bases, which are made of either polished metal or Pyrex. We describe only the results obtained using silver-plated pumping reflectors on metal bases.

The alexandrite rods are 0.13 at. $\% \mathrm{Cr}^{3+}$ concentration, 3-mm in diameter, and $93-120-\mathrm{mm}$ in length, commercially supplied by Allied Signal Corp. or Litton/Airtron. The length of the illuminated area of a rod is $\sim 76 \mathrm{~mm}$. The rod and reflectors are cooled by deionized water at $25^{\circ} \mathrm{C}$ with 10 - and $30-\mathrm{L} / \mathrm{min}$ flow rates, respectively. The laser resonator is of a plane-parallel type with a length of $126 \mathrm{~mm}$. As no mode-selection device is used, the laser is in multimode operation. The crystal $b$ axis of the rod is perpendicular to the plane, which includes the elliptical cylinder foci. In this direction, we find that the pumped rod is thermally unisotropic and is weaker in the direction of the $b$ axis than in the other directions.

\section{Temporal Behavior of the Output Power}

The short-term stability of the laser output is measured with a nanosecond-response $\mathrm{p}-\mathrm{i}-\mathrm{n}$-photodiode. A typical oscilloscope trace [Fig. 3(a)] shows that lasing is continuous but that an oscillatory fluctuation exists. In Fig. 3(a), the averaged laser output $\left(P_{L}\right)$ is $12.8 \mathrm{~W}$, when the supplied power into the lamps $(P)$ equals $3.2 \mathrm{~kW} \times 2$. Furthermore, we find that the dominant frequency of the fluctuation increases with an increase of either $P$ or the output mirror transmission $\Gamma$. This behavior suggests that the fluctuation is caused by the relaxation oscillation. The long-term stability is shown in Fig. 3(b) and is measured at approximately $P_{L} \sim 3 \mathrm{~W}$ using a millisecond-response power meter. When $P_{L}$ is higher than that shown in Fig. 3(b), stability is further improved. The observed long-term peak-to-peak stability $(\sim \pm 3 \%)$ is comparable to stabilities of $\mathrm{Nd}^{3+}$-doped lamp-pumped $\mathrm{cw}$ lasers obtained without the use of stabilization techniques. As the cw lasing of alexandrite is sensitive to disturbance owing to its small gain, the observed laser stability confirms the good stability of the lamp we developed. In both Figs. 3(a) and $3(\mathrm{~b}), P$ is below the critical power level (discussed in Section 2) where the saturation of $P_{L}$ occurs.

\section{Laser Performance}

In Fig. 4, the averaged laser output $P_{L}$ versus $P$ is shown with $\Gamma$ as a parameter. Two combinations of 


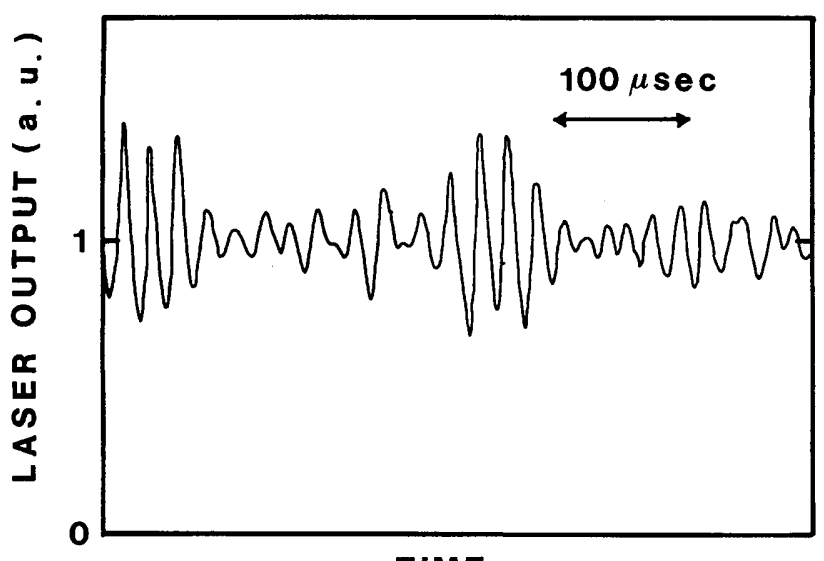

TIME

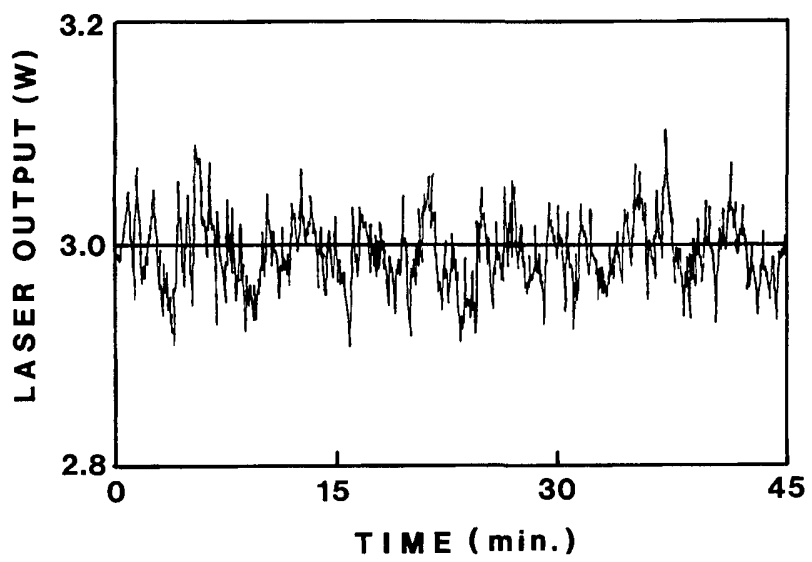

Fig. 3. Typical temporal structure of laser output obtained with output-mirror transmission $\Gamma=1.5 \%$ : (a), spikes resulting from relaxation oscillation measured with a fast-response photodiode; (b), power fluctuation measured with a slow-response power meter.

pumping chambers and rods (Setup A and Setup B) were used. Curves A and B are obtained with Setup $A$; curves $C$ and D are obtained with Setup B. The specifications of Setup A and Setup B are the same but Setup B is less efficient than Setup A because the silver reflector in pump-chamber $B$ tarnishes after $\sim 1000 \mathrm{~h}$ of exposure to open air. In curve $\mathrm{A},(\Gamma=$ $1.5 \%$ ), the pumping-power threshold $P_{\text {th }}$ is $4 \mathrm{~kW}$, and the slope efficiency $\eta_{s}$ is $\sim 0.7 \%$ in a range of $P_{L} 0-12 \mathrm{~W}$.

Taking into account the pumping efficiency of the double-ellipse reflector (usually $60-70 \%$ of that of a single-ellipse reflector ${ }^{4}$ ) and the low concentration of $\mathrm{Cr}^{3+}$ dopant, we estimate the efficiency from pumping to the population inversion in our laser to be $\sim 40 \%$ of that obtained by Samelson et al. ${ }^{2}$ Therefore, for $\Gamma=$ $3 \%, P$ th and $\eta_{s}$ in our laser are expected to be $\sim 6 \mathrm{~kW}$ and $\sim 1 \%$, respectively. The observed values of $P$ th and $\eta_{s}$ for curves $A$ and $B$ are reasonable amounts. This fact confirms that the lamp we developed is as efficient as a conventional AC lamp.

For several of the rods used in our experiments, $P$ th and $\eta_{s}$ (for unsaturated laser power), measured in the same pumping chamber and output coupler, coincide with one another within $\sim \pm 10 \%$. The

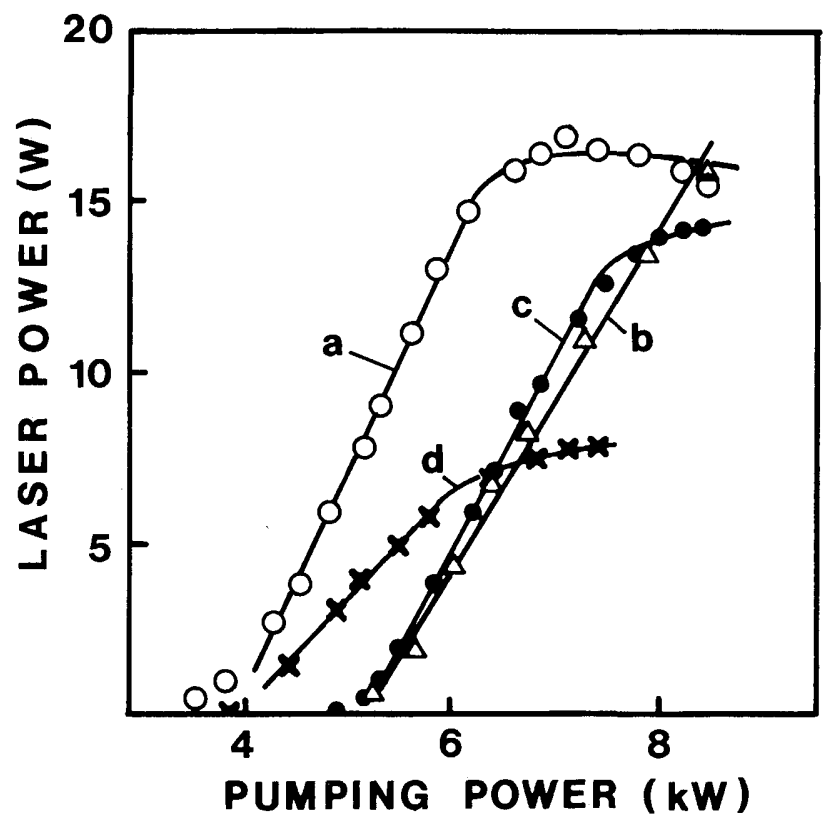

Fig. 4. Laser power versus pumping power in two combinations (Setup A and Setup B) of a pumping chamber and a rod. Using Setup A, we obtained curves A and B, showing output-mirror transmission of $\Gamma=1.5 \%$ and $3.0 \%$, respectively. Using Setup B, we obtained curves $\mathrm{C}$ and $\mathrm{D}$, showing $\Gamma=1.5 \%$ and $0.9 \%$, respectively.

drastic rod dependence see in other studies ${ }^{5}$ is not observed in our experiment. We consider this to owe to the use of relatively recently manufactured rods (having supplier's serial numbers above 970 ) with a constant and dilute concentration of chromium, which minimizes the tolerance and inhomogeneity.

From the above mentioned estimation, we also expected to achieve $\sim 30 \mathrm{~W}$ of laser power by $8-\mathrm{kW}$ pumping. The curves in Fig. 4, however, show that a power saturation prevents this achievement. In spite of a promising near-threshold slope efficiency, we do not obtain a laser power greater than $17 \mathrm{~W}$. The saturation intensity of alexandrite $\left(\sim 150 \mathrm{~kW} / \mathrm{cm}^{2}\right)$ is so high that no saturation was expected in these power levels. However, from additional experiments and analysis, ${ }^{6}$ we find the power saturation is caused by laser reabsorption at the uncooled areas of the laser rod at both ends. Sufficient cooling of the rod avoids the power saturation; however, to our regret, the power in our laser remains limited at $\sim 20$ $\mathrm{W}$, except during short-term operation, because of optical damage to the high-reflection mirrors, which have a $\lambda / 4$ stack of $\mathrm{SiO}_{2}$ and $\mathrm{TiO}_{2}$, that we use. Use of a suitable mirror material or a protective coating, we expect the available laser power will increase to the levels obtained by Samelson et al. ${ }^{2}$

\section{Summary}

We have developed a direct-current driven mercury arc lamp for a cw alexandrite laser. The lamp has much improved stability in comparison with conventional mercury lamps because of special electrodes that we designed that allow us to realize a fully 
vaporized operation. This lamp-pumped $17-\mathrm{W} \mathrm{cw}$ alexandrite laser can be operated with the newly developed lamp for many hours without long-term fluctuations. Although relaxation spikes yet exist, they exhibit an oscillationlike fluctuation in the microsecond range with a constant output [see Fig. 3(b)]. The laser output is not the group of solitary pulses that are often observed in lamp-pumped alexandrite lasers.

The lasing threshold and slope efficiencies of our laser indicate that the newly developed lamp is as efficient as a conventional capillary lamp in pumping of alexandrite. In our cw alexandrite laser, however, the laser power is limited by optical damage and, in some cases, the thermal effects of the rod. Both of these power-limiting factors are more serious in a cw alexandrite laser than in other solid-state lasers of this output level, due to a relatively large circulating power in the laser cavity.

The developed lamp has potential to pump other $\mathrm{Cr}^{3+}$-doped laser materials, such as LiSAF or LiCAF. ${ }^{7}$ A stable DC mercury lamp will open possibilities of various control technique, such as mode locking or injection seeding, for lamp-pumped $\mathrm{cw} \mathrm{Cr}^{3+}$-doped lasers.

\section{References}

1. J.C. Walling, O. G. Peterson, and R. C. Morris, "Tunable cw alexandrite lasers," IEEE J. Quantum Electron. QE-16, 120121 (1980).

2. H. Samelson, J. C. Walling, T. Wernikowski, and D. J. Harter, "Cw arc-lamp-pumped alexandrite laser," IEEE J. Quantum Electron. QE-24, 1141-1150 (1988).

3. K. Torizuka, M. Yamashita, and T. Yabiku, "Continuous wave alexandrite laser pumped by novel mercury arc lamps," in Conference on Lasers and Electro-Optics, Vol. 7 of 1990 Technical Digest Series (Optical Society of America, Washington, D.C., 1990), paper CWF7, p. 254.

4. W. Koechner, Solid-State Laser Engineering, 2nd ed. (SpringerVerlag, Berlin, 1988), pp. 124-125.

5. R. E. Bonanno, D. J. Harter, and O. Montoya, "Enhancement of cw alexandrite laser performance" in Tunable Solid State Lasers, Vol. 5 of 1989 Technical Digest Series (Optical Society of America, Washington, D.C., 1989), paper TUA5, pp. 100-102.

6. K. Torizuka, M. Yamashita, and T. Yabiku, "Thermal effect in a lamp-pumped continuous-wave alexandrite laser," Jpn. J. Appl. Phys. (to be published).

7. S. A. Payne, L. L. Chase, H. W. Newkirk, L. K. Smith, and W. F. Krupke, "LiCaAlF $6: \mathrm{Cr}^{3+}$ : a promising new solid-state laser material," IEEE J. Quantum Electron. 24, 2243-2252 (1988). 